\title{
Konno Procedure for Managing Small Aortic Root during Aortic Valve Replacement Surgery: An Experience of 12 Cases
}

\author{
Ankit Maheshwari, Ravi Gupta, Debmalya Saha, Sayyed Ehtesham Hussain Naqvi, \\ Harpreet Singh Minhas, Muhammad Abid Geelani \\ Department of CTVS, Govind Ballabh Pant Institute of Postgraduate Medical Education \& Research, Delhi, India \\ Email: docankitm05@gmail.com
}

How to cite this paper: Maheshwari, A., Gupta, R., Saha, D., Naqvi, S.E.H., Minhas, H.S. and Geelani, M.A. (2020) Konno Procedure for Managing Small Aortic Root during Aortic Valve Replacement Surgery: An Experience of 12 Cases. World Journal of Cardiovascular Surgery, 10, 24-31. https://doi.org/10.4236/wjcs.2020.102004

Received: January 10, 2020

Accepted: February 21, 2020

Published: February 24, 2020

Copyright $\odot 2020$ by author(s) and Scientific Research Publishing Inc. This work is licensed under the Creative Commons Attribution International License (CC BY 4.0).

http://creativecommons.org/licenses/by/4.0/

(c) (i) Open Access

\begin{abstract}
Background: Small aortic annulus during aortic valve replacement can lead to implanting a smaller sized valve compared to the body surface area thereby causing patient prosthesis mismatch. Various aortic root enlargement techniques have been described depending on anterior or posterior approach. Konno procedure uses anterior approach for aortic root enlargement. In this study, we reviewed results of Konno procedure done from 2011 to 2019 by a single surgeon. Methods: 12 adult patients who underwent aortic valve replacement along with Konno procedure for small aortic root by a single surgeon at a single center between 2011 and 2019 were reviewed. Echocardiographic and demographic data and post-operative data were obtained from medical records. Symptomatic profile was assessed as per New York Heart Association Classification. Intraoperative findings and post-operative period findings were noted. Follow up symptom profile was assessed for these patients. Results: 12 patients underwent Konno procedure between 2011 and 2019 for small aortic root along with valve replacement. The main indication for surgery was aortic stenosis with small aortic annulus, with or without involvement of the mitral valve. Preoperatively, 3 patients had NYHA class II and 9 patients had NYHA class III symptoms. Mean age at operation was 26.42 years, minimum age 10 years, and maximum age 39 years. 3 were females and 9 were males. Mean bypass time was 106.4 minutes and aortic cross clamp time was 80.67 minutes. Mechanical aortic valves were implanted in all patients. Mean post-operative blood loss was $134.2 \mathrm{ml}$ and duration of ventilation before extubation was 14.5 hours. Mean duration of intensive care unit (ICU) stay was 2.83 days and hospital stay was 9.1 days. Mean gradient in the post-operative period was $10.75 \mathrm{~mm} \mathrm{Hg}$. There was no mortality in these 12 patients and no reoperation was needed in the follow up period. Fol-
\end{abstract}


low up in the outpatient department suggested all patients had NYHA class I symptoms and anticoagulation with warfarin adjusted to prothrombin timeInternational normalised ratio. Conclusion: Konno procedure is effective for managing small aortic root as bigger outflow orifice area through the larger valve prosthesis improves ventricular outflow and hence, improves the outcomes.

\section{Keywords}

Konno Operation, Aortic Root Enlargement, Aortic Valve Replacement, Small Aortic Root, Patient Prosthesis Mismatch

\section{Introduction}

Small aortic root and small aortic annulus are often encountered while performing aortic valve replacement. Implanting a smaller sized valve compared to body surface area increases the risk of patient prosthesis mismatch [1]. In previous studies, patient-prosthesis mismatch has been demonstrated to increase left ventricular (LV) work, to reduce LV mass regression, and hence produce symptoms of aortic stenosis [2]. Approach remains controversial, strategies such as aortic root enlargement, supra-annular stented prosthetic valves, stentless bioprosthesis, and sutureless bioprostheses have been proposed [3].

Various procedures have been described for enlarging aortic root which includes both anterior and posterior approach. Konno procedure (aortoventriculoinfundibuloplasty) is an established procedure for enlarging aortic root by anterior approach. Patients with larger body surface area (BSA) will require higher flow rates across the valve (cardiac output) than those with a smaller BSA. Knowledge of the patient's BSA and the effective orifice area (EOA) of prosthesis give an idea about the minimum valve size needed. Patient-specific factors such as age and activity level can be considered for calculating cardiac output demand; young people with active work profile will require larger prosthesis for a higher cardiac demand [4].

Since implanting an undersized valve or not intervening on small aortic root can worsen outcome due to increased preload, Konno procedure is extremely useful. Hence, we performed this study to review the cases in which Konno procedure was done.

\section{Material and Methods}

\subsection{Patient Characteristics}

Between 2011 and 2019, 12 patients underwent Konno procedure along with aortic valve replacement with mechanical aortic valve prostheses were performed in our hospital (Table 1). Of these 12 patients, 11 underwent aortic valve replacement alone and one patient underwent mitral valve replacement along with aortic valve replacement. Among them, 3 were females, and 9 were males, 
Table 1. Preoperative profile.

\begin{tabular}{ll}
\hline Mean age & 26.42 years \\
Males & 9 \\
Females & 3 \\
Mean Body surface area & 1.84 \\
Height (mean in centimeters) & 170.92 \\
Weight (mean in kilograms) & 72.25 \\
Echocardiography & \\
1) Aortic annulus diameter (mean in centimetres) & 1.89 \\
2) Pre-operative echocardiography gradient (mean in mmHg) & 87.75 \\
a) Peak gradient & \\
b) Mean gradient & \\
3) Left ventricular ejection fraction (mean in percentage) & 55.5 \\
4) Aortic valve disease etiology (number of patients) & $60 \%$ \\
a) Rheumatic heart disease & 8 \\
b) Bicuspid aortic valve & 3 \\
c) Calcific + bicuspid aortic valve & 1 \\
NYHA class of symptoms (number of patients) & \\
I & - \\
II & 3 \\
III & 9 \\
IV & - \\
\hline
\end{tabular}

with a mean age of 26.42 years, minimum age of 10 years and maximum age 39 years. Mean body surface area was $1.84 \mathrm{~m}^{2}$. Preoperative clinical features are shown in Table 1. Symptoms at admission included dyspnoea with angina in 2 patients, angina in 10 patients, and no patient had history of syncope or arrythmia. Mean preoperative New York Heart Association (NYHA) class was 2.6. Since all patients were of age less than 40 years, coronary angiography was not done as coronary artery disease was rare in this age group. Aetiology for aortic stenosis was bicuspid aortic valve alone in 3 patients, bicuspid aortic valve with calcification in 1 patient and rheumatic aetiology in 8 patients, all patients gave informed consent to publication of their data. Preoperative evaluation included hemogram, kidney and liver function tests, chest radiographs, electrocardiogram and echocardiography. A complete M-mode, two-dimensional, and Doppler evaluation was performed. LV ejection fraction, aortic annulus diameter and aortic valve morphology were noted. The peak and mean gradients across the aortic valve as well as across the prostheses were calculated according to the modified Bernoulli equation. Mean aortic annulus size was $1.89 \mathrm{~cm}$, while the peak gradient (mean in $\mathrm{mmHg}$ ) was 87.75 and mean gradient (mean in $\mathrm{mmHg}$ ) 55.5. Echocardiographic evaluation was repeated before discharge. Symptom profile was evaluated at follow up.

\subsection{Procedure}

Approach for all patients was by standard median sternotomy. Pericardial patch 
was harvested and fixed with glutaraldehyde. Heparinisation was done with unfractionated heparin at a dose of $4 \mathrm{mg} / \mathrm{kg}$ body weight. The ascending aorta was cannulated, venae cavae was cannulated individually for all patients and looped with cotton tape.

The aortic cross clamp was applied, cardioplegia was is infused in the aortic root. Del Nido cardioplegia solution was used for all patients. A left ventricular vent was placed through the right superior pulmonary vein. In one case left atrium was opened for mitral valve replacement, anterior mitral leaflet was excised, mitral valve prosthesis was implanted and fixed. Looped venae cavae were snugged. A vertical aortotomy was done. The incision carried out onto right ventricular outflow tract. The incision was carried out taking care to avoid injury to the pulmonary valve. The incision was kept to the left of the origin of right coronary artery. The aortic valve leaflets were excised. Incision was made across the aortic annulus extending to superior part of the infundibular septum. Care was taken to avoid injury to the conduction tissue. Pericardial patch was sutured to the edges of the septal incision with interrupted pledgeted mattress sutures (Figure 1). Bileaflet mechanical valves were used for insertion in all patients. It was ensured that the size of the valve was appropriate according to body surface area avoiding patient prosthesis mismatch. Anteriorly, the valve sutures were passed through the pericardial patch (Figure 2). The superior portion of the patch was used to close the ascending aorta. The right ventricular free wall was augmented with pericardial patch (Figure 3). Deairing was done, and the aortic

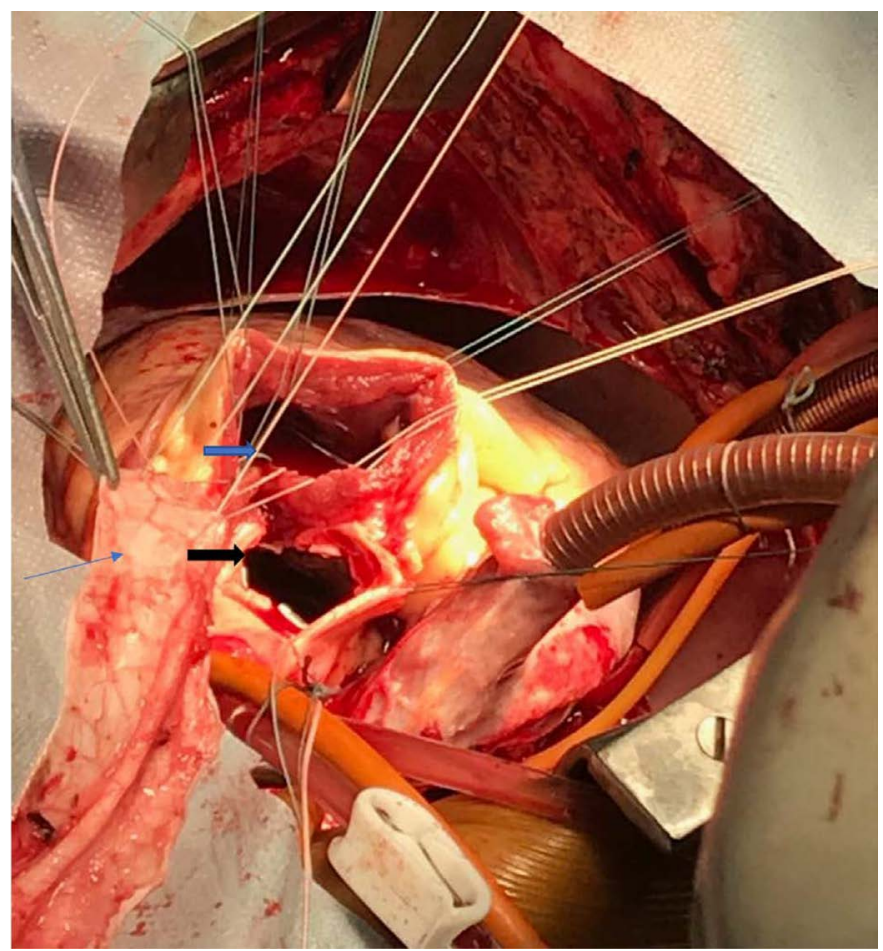

Figure 1. Pericardial patch (fine blue arrow) sutured to the edges of the septal incision with interrupted pledgeted mattress sutures. Enlarged aortic root (black arrow). Right ventricular outflow tract (broad blue arrow). 


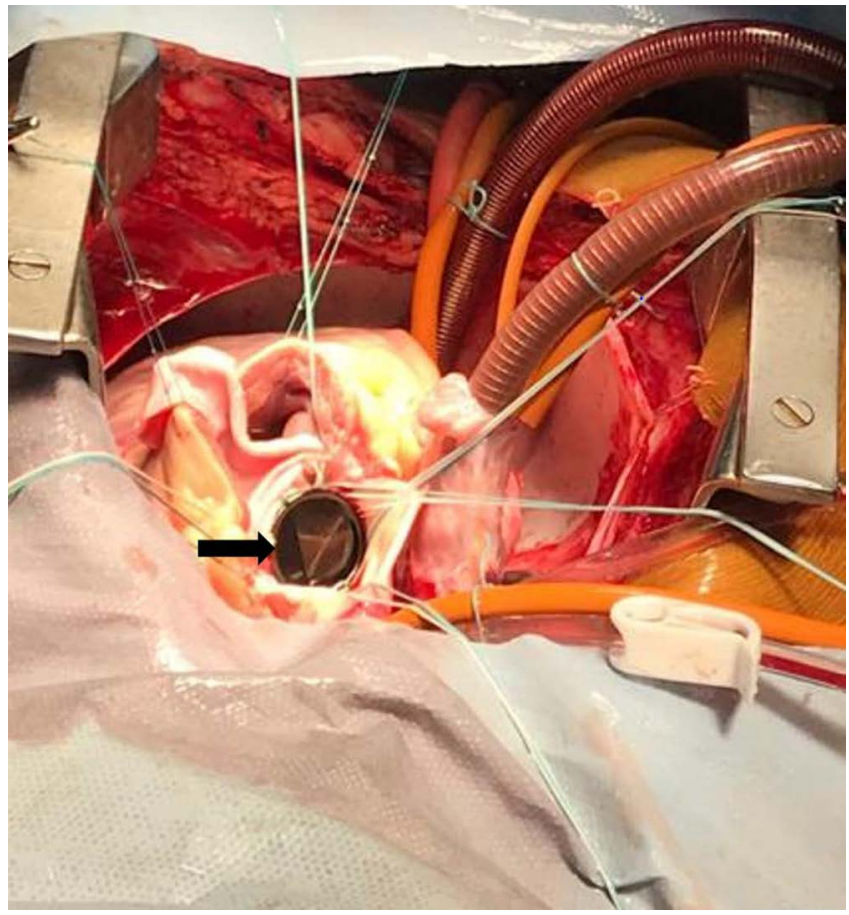

Figure 2. Bileaflet mechanical valve (black arrow) implanted in enlarged aortic root. Anteriorly, the valve sutures are passed through the pericardial patch.

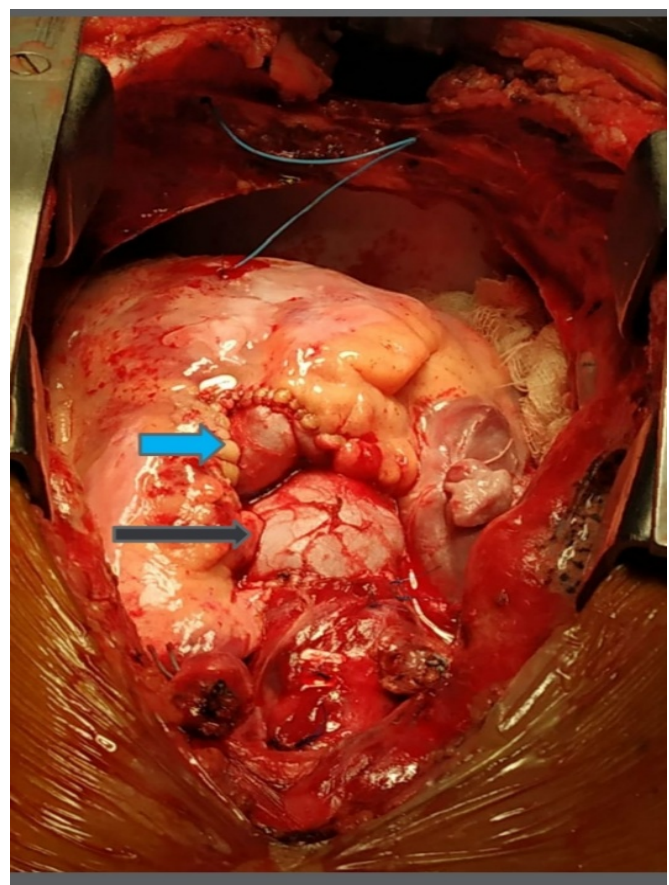

Figure 3. The superior portion of pericardial patch used to close the ascending aorta (black arrow). The right ventricular free wall was augmented with pericardial patch (blue arrow).

clamp was removed. After weaning from cardiopulmonary bypass with inotropic support, left ventricular vent was removed and bicaval decannulation was done. Cardioplegia cannula was removed. Reversal of heparin was done with prota- 
mine. Aortic cannula was removed, pacing wire and mediastinal drains were inserted. After haemostasis was achieved, incision was closed in layers.

\section{Results}

12 patients underwent Konno procedure between 2011 and 2019 for small aortic root along with valve replacement (Table 2). The main indication for surgery was aortic stenosis with small aortic annulus, with or without involvement of the mitral valve. Mean bypass time was 106.4 minutes and aortic cross clamp time was 80.67 minutes. Mechanical aortic valves were implanted in all patients. SJM regent series aortivc valve prosthesis was implanted in one patient, Medtronics ATS MEDICAL AP 360 was implanted in 6 patients and Carbomedics valve was implanted in 5 patients. Mean post-operative blood loss was $134.2 \mathrm{ml}$ and duration of ventilation before extubation was 14.5 hours. All patients remained haemodynamically stable during ICU and hospital stay. Laboratory investigations remained within normal limits and were no post-operative arrythmia. Surgical site remained healthy in all patients. Mean duration of ICU stay was 2.83 days and hospital stay was 9.1 days. Mean gradient in the post-operative period was $10.75 \mathrm{mmHg}$. Indexed effective orifice area (effective orifice area/body surface area) was 1.11 (mean in $\mathrm{cm}^{2} / \mathrm{m}^{2}$ ). There was no mortality in these 12 patients and no reoperation was needed in the follow up period. All patients were started on anticoagulation therapy, low molecular weight heparin and oral

Table 2. Intraoperative and postoperative data.

\begin{tabular}{lc}
\hline Valve inserted (number of patients) & \\
1. St Jude Medical Regent series & 1 \\
Size 21 & 3 \\
2. Medtronics ATS MEDICAL AP 360 & 3 \\
Size 18 & \\
Size 20 & 1 \\
3. Carbomedics & 4 \\
Size 21 & \\
Size 23 & 1 \\
Additional procedure & 1.11 \\
1. Mitral valve replacement & 106.4 \\
EOA/BSA (cm $2 / \mathrm{m}^{2}$ ) & 80.67 \\
Cardiopulmonary bypass time (mean in minutes) & 134.2 \\
Aortic cross clamp time (mean in minutes) & 2.83 \\
Blood loss in first 24 hours (mean in mL) & 8.5 \\
Mean ICU stay (days) & none \\
Mean Hospital stay (days) & 10.75 \\
Mortality & \\
Post-operative mean gradient (mean in mmHg) & \\
\hline
\end{tabular}


warfarin was given till the target INR of 2.0 was achieved. Warfarin dose was then adjusted in the follow up period keeping a target INR of 2.0. Follow up in the outpatient department suggested all patients had NYHA class I symptoms.

\section{Discussion}

The term "prosthesis patient mismatch" as coined in 1978 by Rahimtoola is used to describe that stenosis of a valve may not be relieved by a prosthesis [1]. This is evident by laws of physics as Poiseuille's law states inverse relationship between resistance and fourth power of radius. Increase in diameter of prosthesis gives benefit of increased effective orifice area. Implanting a valve with larger diameter leads to reduced resistance and improved flow across the valve. Maximum increase in LV outflow size should not compromise with operative risk.

Depending on the manufacturer of the valve, effective orifice area varies with size. Prosthesis patient mismatch (PPM) is avoided if iEOA (EOA/BSA) $>0.85$ $\mathrm{cm}^{2} / \mathrm{m}^{2}$. EOA/BSA of 0.65 to $0.85 \mathrm{~cm}^{2} / \mathrm{m}^{2}$ leads to moderate PPM. EOA/BSA < $0.65 \mathrm{~cm}^{2} / \mathrm{m}^{2}$ is considered severe PPM. Hence for calculating appropriate size for valve, patient's body surface area from patient's weight and height is calculated. BSA is multiplied by $0.85 \mathrm{~cm}^{2} / \mathrm{m}^{2}$, this value will be minimal EOA for the prosthesis to be implanted. This is verified by reference EOA provided by the manufacturer for the model and size of prosthesis selected. The surgeon should try to implant prosthesis with a larger EOA. PPM is a strong and independent predictor of short-term mortality among patients undergoing AVR, and its impact is related both to its degree of severity and the status of left ventricular function [5].

Nicks (1970) and Manouguian (1979) each described techniques of patch enlargement of the posterior aspect of the aortic annulus. Konno described a more extensive procedure of aortoventriculoplasty of the anterior aspect of the aortic annulus in 1975. More recently, Otaki described a method of patch enlargement of both the posterior and anterior aortic annulus (1997). Each of these has their advantages and disadvantages. Previous studies have been done debating the benefits and risks of aortic root enlargement and their association of these procedures with mortality. Castro et al. performed root enlargement in 114 out of 657 patients with Aortic valve replacement. However, they demonstrated how aortic root enlargement can be performed with minimal added risk relative to standard aortic valve replacement. Their study showed prolongation of the cardiopulmonary bypass time beyond 120 minutes was the only risk factor for increased mortality [6].

Kulik et al. showed that for patients with small aortic roots, aortic root enlargement at the time of Aortic valve replacement is a safe procedure that reduces postoperative gradients and the incidence of prosthesis-patient mismatch, but this procedure does not appreciably improve long-term clinical outcomes. However, the procedure was associated with better freedom from late congestive heart failure [7]. In an analysis done by Rodolfo V. Rocha et al., aortic root en- 
largement was not associated with increased risk of mortality or adverse events. They suggested aortic root enlargement is safe adjunct to aortic valve replacement [8]. Konno procedure is a well documented in the past. Our study demonstrates that the procedure is associated with a significant decline in LVOTO gradient, stabilization of left ventricular function, and improvement in functional class.

In our series, all patients undergoing Konno procedure with aortic valve replacement had good post-operative outcomes in terms of mean gradient and no mortality. Also, all the patients had symptoms in NYHA class I in the post-operative period. The limitation of our study is that there is no long-term follow-up data. However, based on our experience, Konno procedure is a safe technique and we encourage using this procedure to avoid mismatch.

\section{Conclusion}

In our study, we find Konno procedure as an effective and safe method to enlarge aortic root. Implanting a larger size prosthesis avoids patient prosthesis mismatch and leads to improved symptom profile on follow up.

\section{Conflicts of Interest}

The authors declare no conflicts of interest regarding the publication of this paper.

\section{References}

[1] Rahimtoola, S.H. (1978) The Problem of Valve Prosthesis-Patient Mismatch. Circulation, 58, 20-24. https://doi.org/10.1161/01.CIR.58.1.20

[2] Pibarot, P., Dumesnil, J.G., Lemieux, M., Cartier, P., Metras, J. and Durand, L.G. (1998) Impact of Prosthesis-Patient Mismatch on Hemodynamic and Symptomatic Status, Morbidity and Mortality after Aortic Valve Replacement with a Bioprosthetic Heart Valve. The Journal of Heart Valve Disease, 7, 211-218.

[3] Freitas-Ferraz, A.B., et al. (2019) Aortic Stenosis and Small Aortic Annulus. Circulation, 139, 2685-2702. https://doi.org/10.1161/CIRCULATIONAHA.118.038408

[4] Fullerton, D.A. (1998) Mastery of Cardiothoracic Surgery. Chapter 48: Aortic Valve Replacement. 3rd Edition, Lippincott Williams \& Wilkins, Philadelphia, p. 455.

[5] Blais, C., et al. (2003) Impact of Valve Prosthesis-Patient Mismatch on Short-Term Mortality after Aortic Valve Replacement. Circulation, 108, 983-988. https://doi.org/10.1161/01.CIR.0000085167.67105.32

[6] Castro, L.J., Arcidi, J.M.J., Fisher, A.L., et al. (2002) Routine Enlargement of the Small Aortic Root: A Preventive Strategy to Minimize Mismatch. The Annals of Thoracic Surgery, 74, 31-36. https://doi.org/10.1016/S0003-4975(02)03680-9

[7] Kulik, A., et al. (2008) Enlargement of the Small Aortic Root during Aortic Valve Replacement: Is There a Benefit? The Annals of Thoracic Surgery, 85, 94-100. https://doi.org/10.1016/j.athoracsur.2007.07.058

[8] Rocha, R.V., et al. (2018) Surgical Enlargement of the Aortic Root Does Not Increase the Operative Risk of Aortic Valve Replacement. Circulation, 137, 1585-1594. https://doi.org/10.1161/CIRCULATIONAHA.117.030525 